\title{
Integrated nanozymes with nanoscale proximity for in vivo neurochemical monitoring in living brains
}

Hanjun Cheng ${ }^{1}$, Lei Zhang ${ }^{1}$, Jian $\mathrm{He}^{2}$, Wenjing Guo ${ }^{1}$, Zhengyang Zhou ${ }^{2} *$, Xuejin Zhang ${ }^{1, *}$, Shuming $\mathrm{Nie}^{1,3, *}$ and Hui Wei ${ }^{1,4, *}$

${ }^{1}$ College of Engineering and Applied Sciences, Nanjing National Laboratory of Microstructures, Nanjing University, Nanjing, Jiangsu, 210093, China .

${ }^{2}$ Department of Radiology, Nanjing Drum Tower Hospital, the Affiliated Hospital of Nanjing University Medical School, Nanjing, Jiangsu, 210008, China.

${ }^{3}$ Department of Biomedical Engineering, Emory University, Atlanta, Georgia, 30322, USA.

${ }^{4}$ Collaborative Innovation Center of Chemistry for Life Sciences, State Key Laboratory of Analytical Chemistry for Life Science, Nanjing University, Nanjing, Jiangsu, 210093, China.

Email: weihui@nju.edu.cn; Tel: +86-25-83593272; Fax: +86-25-83594648; Web: weilab.nju.edu.cn

Email: snie@emory.edu; Tel: +1-404-712-8595; Fax: +1-404-727-3567

Email: xuejinzh@nju.edu.cn; Tel: +86-25-83594683

Email: zyzhou@nju.edu.cn; Tel: +86-25-68182222 


\section{Table of contents}

\section{Experimental Section}

Figure S1. The INAzyme-based integrative fluorescent sensing platform for continuous in vivo measurement of neurochemicals (glucose as an example in the current study) in living rats.

Figure S2. XRD patterns of different ZIF-8 samples: simulated (black curve), ZIF-8 (red curve), hemin@ZIF-8 (blue curve), and the INAzyme (pink curve), respectively.

Figure S3. TEM images and corresponding element mapping for ZIF-8 (a), hemin@ZIF-8 (b) and the INAzyme (c).

Figure S4. Bright field (left) and the corresponding fluorescent (right) images of GOx-FITC/hemin@ZIF-8 $\left(\lambda_{\mathrm{ex}}=436 \mathrm{~nm}\right)$.

Figure S5. DLS of ZIF-8 and ZIF-8 based nanozymes.

Figure S6. Typical photograph of $1 \mathrm{~mL}$ of $0.10 \mathrm{M}$ Tris- $\mathrm{HCl}$ buffer (pH 7.0) containing (1) $200 \mu \mathrm{M}$ $\mathrm{H}_{2} \mathrm{O}_{2}$, (2) $2 \mathrm{mM}$ ABTS, (3) $200 \mu \mathrm{M} \mathrm{H}_{2} \mathrm{O}_{2}+2$ mM ABTS, (4) $200 \mu \mathrm{M} \mathrm{H}_{2} \mathrm{O}_{2}+2 \mathrm{mM}$ ABTS +100 $\mu \mathrm{g} / \mathrm{mL}$ ZIF-8, and (5) $\mathrm{H}_{2} \mathrm{O}_{2}+2$ mM ABTS + $100 \mu \mathrm{g} / \mathrm{mL}$ hemin @ZIF-8.

Figure S7. Time-dependent absorption changes at $421 \mathrm{~nm}$ by adding hemin alone $(\bullet)$ or hemin@ZIF-8 nanozymes ( $\square$ ) into $0.10 \mathrm{M}$ Tris- $\mathrm{HCl}$ buffer (pH 7.0) containing $300 \mu \mathrm{M} \mathrm{H}_{2} \mathrm{O}_{2}$ and $2 \mathrm{mM}$ ABTS. The final concentration of hemin for each solution was calculated to be $2 \mu \mathrm{M}$.

Figure S8. UV-visible spectra of free hemin dissolved in DMSO (black curve) or water (red curve), ZIF-8 (blue curve) and hemin@ZIF-8 (green curve) aqueous solutions.

Figure S9. Normalized catalytic activities of hemin@ZIF-8 after recycling.

Figure S10. Kinetic plots for time-dependent $\Delta \mathrm{A}_{421}$ of $0.10 \mathrm{M}$ Tris-HCl buffer (pH 7.0) containing 250 $\mu \mathrm{M}$ glucose, $2 \mathrm{mM}$ ABTS, and different concentrations of the INAzyme. The final concentrations of the INAzyme were indicated in the figure.

Figure S11. Typical photograph of $1 \mathrm{~mL}$ of $0.10 \mathrm{M}$ Tris- $\mathrm{HCl}$ buffer (pH 7.0) containing (1) $200 \mu \mathrm{M}$ glucose, (2) $2 \mathrm{mM}$ ABTS, (3) $200 \mu \mathrm{M}$ glucose + 2 mM ABTS, (4) $200 \mu \mathrm{M}$ glucose + $2 \mathrm{mM}$ ABTS + $200 \mu \mathrm{g} / \mathrm{mL}$ ZIF-8, (5) $200 \mu \mathrm{M}$ glucose $+2 \mathrm{mM} \mathrm{ABTS} \mathrm{+} 200 \mu \mathrm{g} / \mathrm{mL}$ hemin@ZIF-8, (6) $200 \mu \mathrm{M}$ 
glucose + 2 mM ABTS + $200 \mu \mathrm{g} / \mathrm{mL}$ GOx@ZIF-8, and (7) $200 \mu \mathrm{M}$ glucose + 2 mM ABTS + 200 $\mu \mathrm{g} / \mathrm{mL}$ the INAzyme.

Figure S12. (a) Kinetic plots of time-dependent $\Delta \mathrm{A}_{421}$ of $0.10 \mathrm{M}$ Tris- $\mathrm{HCl}$ buffer (pH 7.0) containing $200 \mu \mathrm{M}$ glucose, $2 \mathrm{mM}$ ABTS, and $200 \mu \mathrm{g} / \mathrm{mL}$ fleshly prepared (ש) or 2 month-old (•) INAzymes. (b) Normalized cascade enzymatic activity of fleshly prepared and 2 month-old INAzymes.

Figure S13. Normalized fluorescent intensity of leaked PPIX from GOx-FITC/PPIX@ZIF-8 over 2 months.

Figure S14. Normalized catalytic activity of free GOx + hemin@ZIF-8 and INAzyme, showing the encapsulation of GOx within INAzyme had little effect on its catalytic activity.

Figure S15. Nanoscale proximity effects on the catalytic activities of LOx/hemin@ZIF-8 INAzyme.

Figure S16. Nanoscale proximity effects on the catalytic activities of invertase/GOx/hemin@ZIF-8 INAzyme.

Figure S17. In vitro detection of glucose with the INAzyme.

Figure S18. Selective detection of glucose with the INAzyme.

Figure S19. Typical fluorescence intensity-time curve for glucose, the mixtures of glucose and AA, and the mixtures of glucose and lactate (concentrations indicated in the figure).

Figure S20. Typical fluorescence intensity-time curve for the microdialysate monitored on the integrative sensing platform with GOx@ZIF-8-immobilized microfluidic chip.

Table S1. Zeta potential of ZIF-8 and ZIF-8-based nanozymes.

\section{References}




\section{Experimental Section}

Chemicals and Materials. Zinc acetate, 2-methylimidazole and glucose were purchased from Nanjing Chemical Co. (Nanjing, China). 2,2'-azino-bis(3-ethylbenzo-thiazoline-6-sulfonic acid) diammonium salt (ABTS), hemin, fluorescein isothiocyanate isomer I (FITC), sodium ascorbate (AA), dopamine (DA), 3,4-dihydroxyphenylacetic acid (DOPAC), uric acid (UA), 5-hydroxytryptamine (5-HT), L-(+)-lactate acid, Ampliflu Red and GOx (E.C. 1.1.3.4, from Aspergillus niger) were obtained from Sigma and used as received. Other chemicals were of at least analytical reagent and were used as received. The stock solution of Ampliflu Red was prepared by dissolving Ampliflu Red into dimethylsulfoxide (DMSO). Artificial cerebrospinal fluid (aCSF) at pH 7.4 containing $\mathrm{NaCl}(126 \mathrm{mM})$, $\mathrm{KCl}(2.4 \mathrm{mM}), \mathrm{KH}_{2} \mathrm{PO}_{4}(0.5 \mathrm{mM}), \mathrm{MgCl}_{2}(0.85 \mathrm{mM}), \mathrm{NaHCO}_{3}(27.5 \mathrm{mM}), \mathrm{Na}_{2} \mathrm{SO}_{4}(0.5 \mathrm{mM})$, and $\mathrm{CaCl}_{2}(1.1 \mathrm{mM})$ was used. All aqueous solutions used in the experiments were prepared with deionized water (18.2 $\mathrm{M} \Omega \cdot \mathrm{cm}$, Millipore).

Labeling GOx with fluorescent FITC. $100 \mu \mathrm{L}$ of $1 \mathrm{mg} / \mathrm{mL}$ FITC in dry DMSO was slowly added into $2 \mathrm{~mL}$ of $2 \mathrm{mg} / \mathrm{mL}$ GOx in $0.10 \mathrm{M}$ sodium carbonate (pH 9.0) solution. The mixed solution was gently stirred at $4{ }^{\circ} \mathrm{C}$ in the dark for at least $8 \mathrm{~h}$. The reaction was quenched by the addition of ammonium chloride to a final concentration of $50 \mathrm{mM}$. Finally, the free FITC was completely removed by dialysis at $4{ }^{\circ} \mathrm{C}$ in the dark for $24 \mathrm{~h}$. The prepared FITC-conjugated GOx was thus denoted as GOx-FITC.

Instrumentation. Powder X-ray diffraction (XRD) data were collected on an ARL SCINTAG X'TRA diffractometer by using $\mathrm{Cu} \mathrm{K} \alpha$ radiation (Thermo). Transmission electron microscopy (TEM) was performed on a JEOL 2100F transmission electron microscope at an acceleration voltage of $200 \mathrm{kV}$. The X-ray energy-dispersive spectrometry (EDS) and element mapping analysis were carried out with an EDS detector on the JEOL 2100F transmission electron microscope. UV-visible absorption spectra were collected on a UV-visible spectrophotometer with a 0.2-cm quartz cell (Beijing Purkinje General Instrument Co. Ltd., China) and fluorescent spectra were obtained on a Hitachi F-4600 fluorescent spectrometer (Japan). The fluorescent images of GOx-FITC/hemin@ZIF-8 were taken on a fluorescent microscopy. 


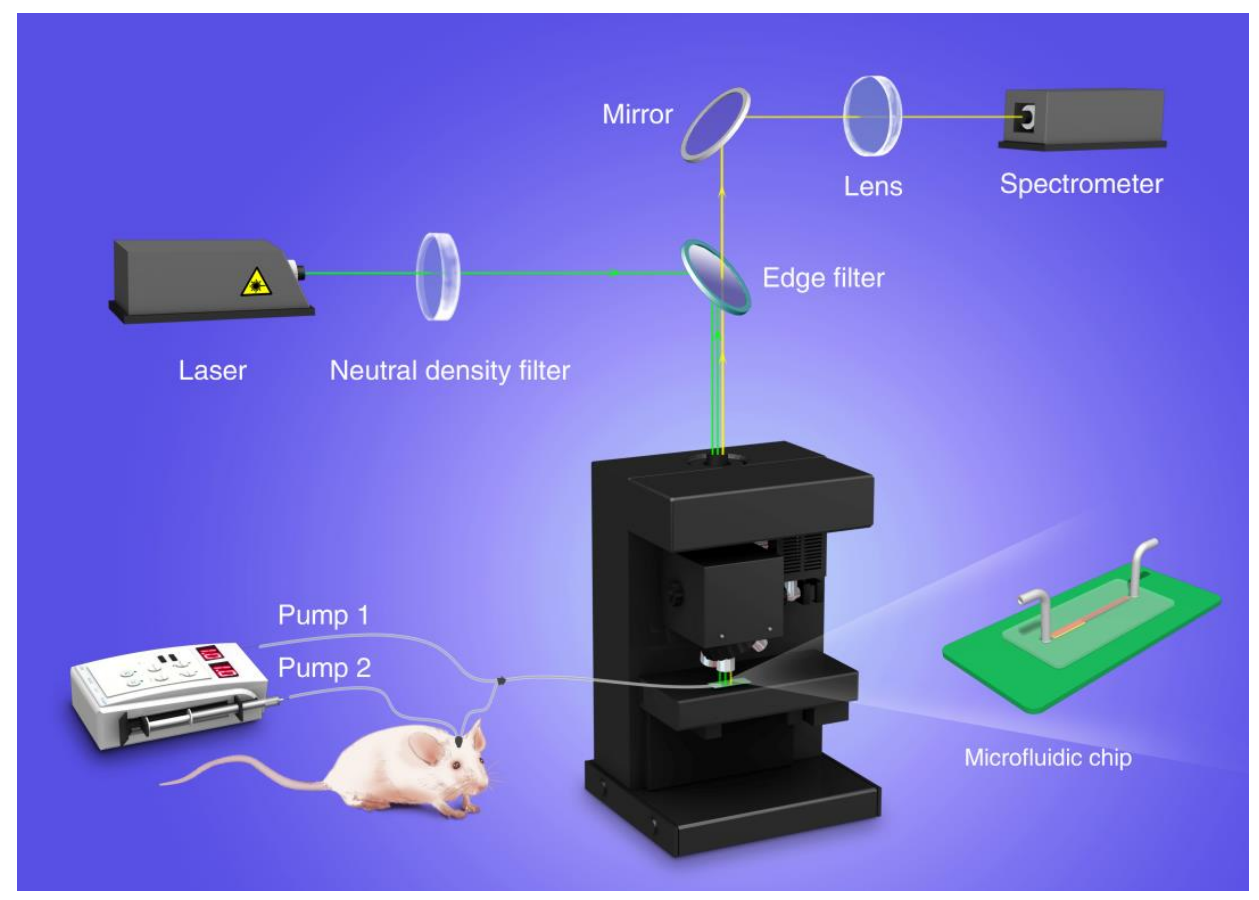

Figure S1. The INAzyme-based integrative fluorescent sensing platform for continuous in vivo measurement of neurochemicals (glucose as an example in the current study) in living rats. The platform mainly consisted of three parts: (a) the microdialysis device, which included two pumps with syringes, a microdialysis probe (inserted in the rat's brain), and a T-junction, connected with tubing; (b) the fluorescent microscope, which included the excitation laser and a spectrometer; and (c) the microfluidic chip, which was connected with the microdialysis device via the tubing and had the INAzyme immobilized in its microchannel. The $532 \mathrm{~nm}$ laser through the microscope was focused onto the sample within the microchannel with a distance of $2 \mathrm{~mm}$ far from the outlet. The resultant fluorescent spectra of the sample were continuously collected. 


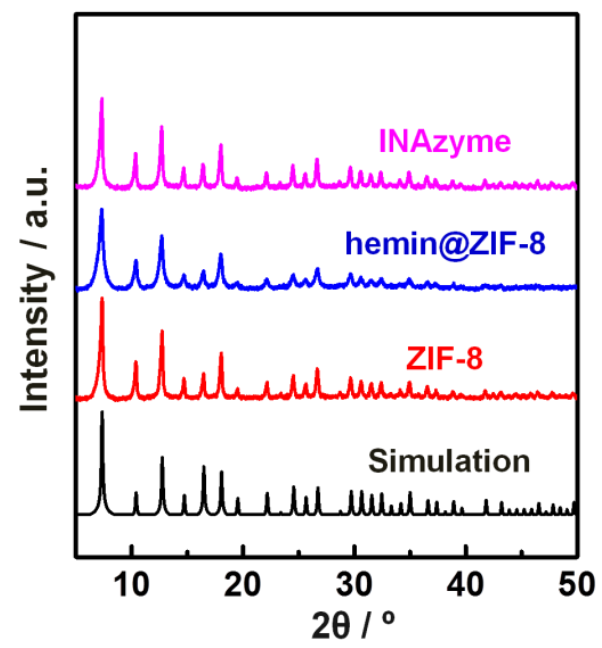

Figure S2. XRD patterns of different ZIF-8 samples: simulated (black curve), ZIF-8 (red curve), hemin@ZIF-8 (blue curve), and the INAzyme (pink curve), respectively. 

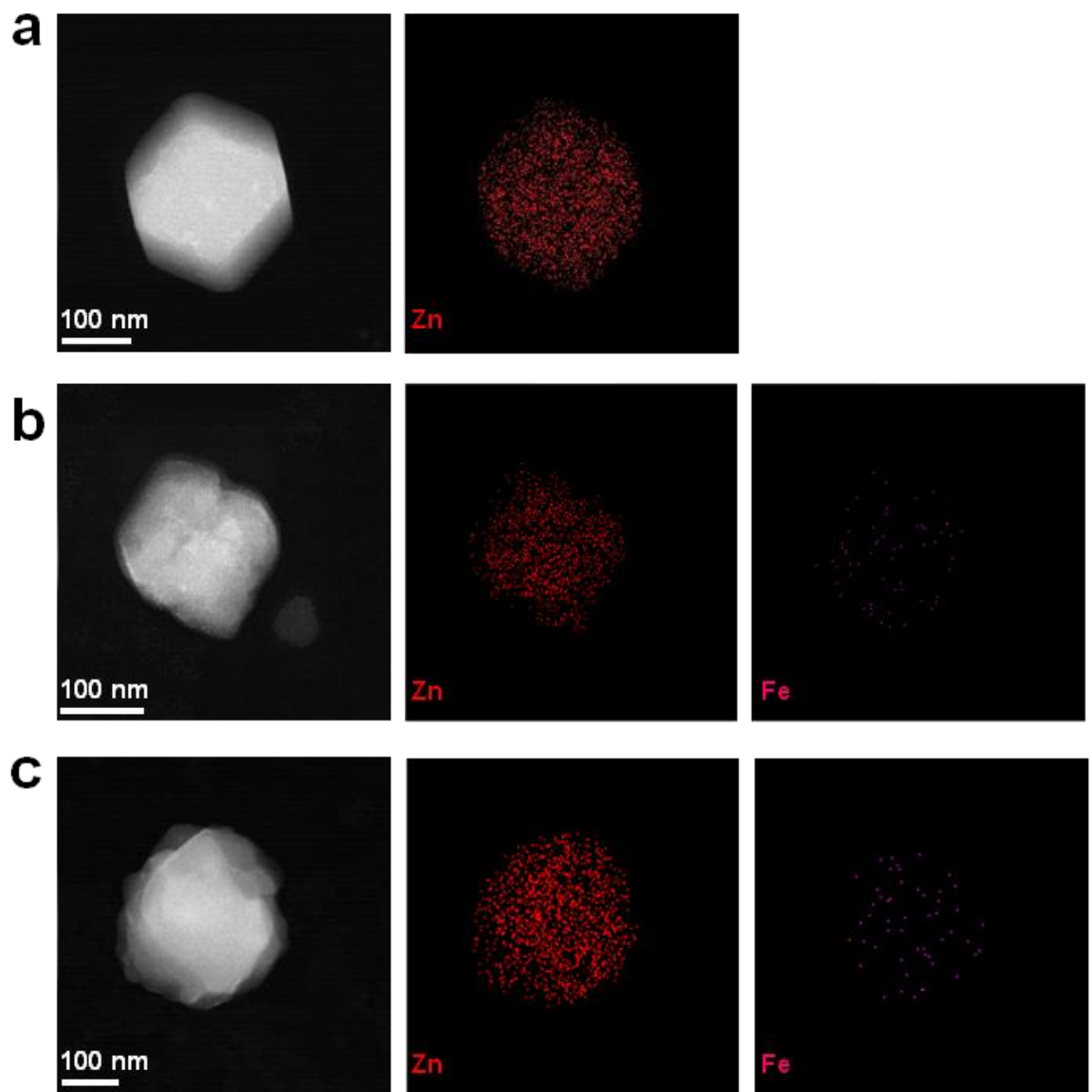

Figure S3. TEM images and corresponding element mapping for ZIF-8 (a), hemin@ZIF-8 (b) and the INAzyme (c). The $\mathrm{Zn}$ signals were from the ZIF-8 matrices while the Fe signals were from the encapsulated hemin.

The percentage of $\mathbf{S}$ element in the INAzyme. For each chain of GOx, it has a molecular weight of 63980. There are 3 Cys and 15 Met residues in a chain of GOx. Thus the mass percentage of S element in GOx, can be calculated as $\mathrm{S} \%=(3+15) * 32 / 63980=0.90 \%$. The mass percentage of $\mathrm{S}$ element in the INAzyme should be much less than $0.90 \%$. 


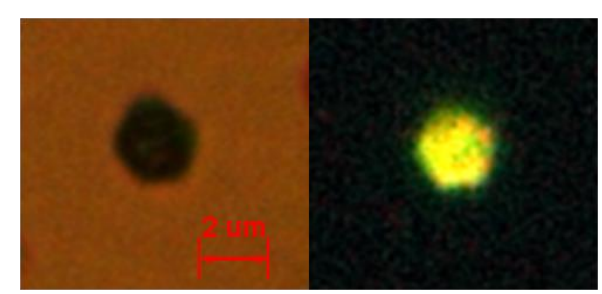

Figure S4. Bright field (left) and the corresponding fluorescent (right) images of GOx-FITC/hemin@ZIF-8 $\left(\lambda_{\mathrm{ex}}=436 \mathrm{~nm}\right)$.

The bright lime green fluorescence from the GOx-FITC/hemin@ZIF-8 nanoparticles confirmed the successful encapsulation of GOx-FITC into the ZIF-8 frameworks. Note, the observed fluorescent image was from the GOx-FITC/hemin@ZIF-8 assembly instead of single nanoparticles due to the limited resolution of the fluorescence microscope used. 

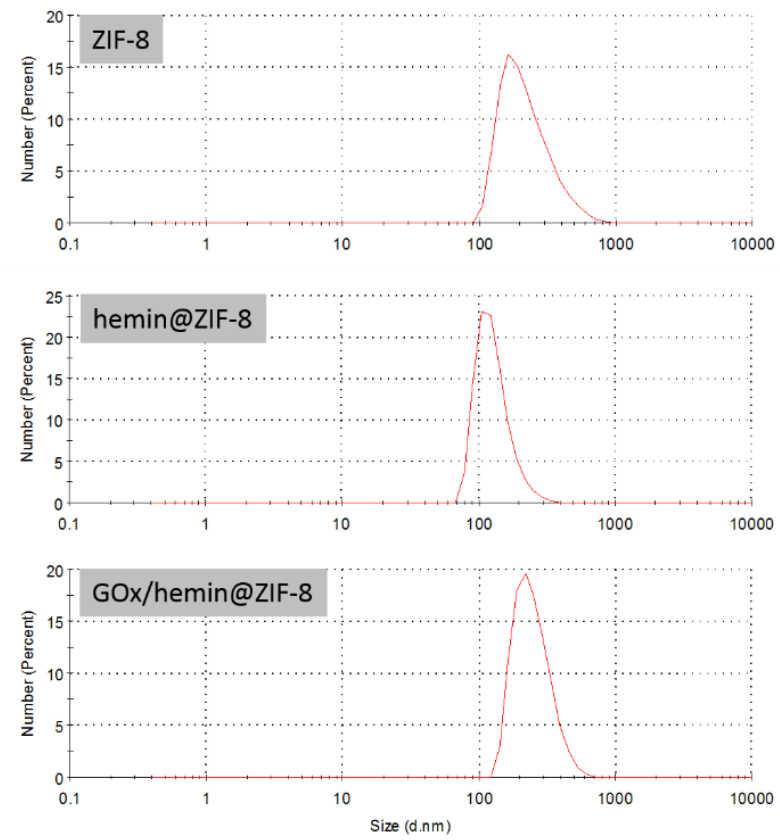

Figure S5. DLS of ZIF-8 and ZIF-8 based nanozymes. 


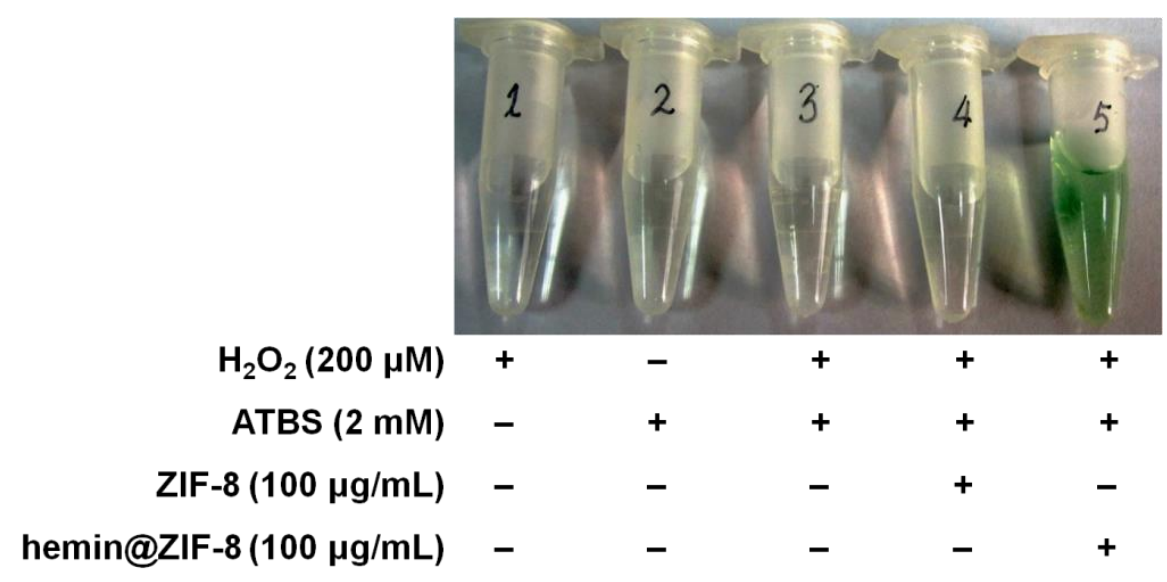

Figure S6. Typical photograph of $1 \mathrm{~mL}$ of $0.10 \mathrm{M}$ Tris- $\mathrm{HCl}$ buffer (pH 7.0) containing (1) $200 \mu \mathrm{M}$ $\mathrm{H}_{2} \mathrm{O}_{2}$, (2) $2 \mathrm{mM}$ ABTS, (3) $200 \mu \mathrm{M} \mathrm{H}_{2} \mathrm{O}_{2}+2 \mathrm{mM} \mathrm{ABTS}$, (4) $200 \mu \mathrm{M} \mathrm{H}_{2} \mathrm{O}_{2}+2 \mathrm{mM}$ ABTS +100 $\mu \mathrm{g} / \mathrm{mL}$ ZIF-8, and (5) $\mathrm{H}_{2} \mathrm{O}_{2}+2 \mathrm{mM} \mathrm{ABTS}+100 \mu \mathrm{g} / \mathrm{mL}$ hemin@ZIF-8. 


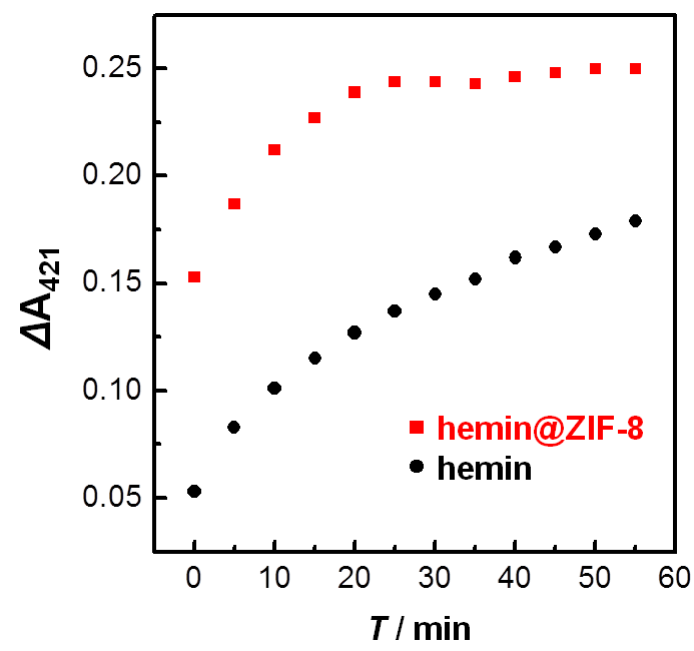

Figure S7. Time-dependent absorption changes at $421 \mathrm{~nm}$ by adding hemin alone (•) or hemin @ZIF-8 nanozymes ( $\square$ ) into $0.10 \mathrm{M}$ Tris- $\mathrm{HCl}$ buffer (pH 7.0) containing $300 \mu \mathrm{M} \mathrm{H}_{2} \mathrm{O}_{2}$ and $2 \mathrm{mM}$ ABTS. The final concentration of hemin for each solution was calculated to be $2 \mu \mathrm{M}$.

\section{The underestimated enhancement of peroxidase mimicking activity for hemin@ZIF-8 compared} with that for free hemin. The underestimated enhancement was due to the following facts: first, the yield of hemin encapsulation within hemin@ZIF-8 framework was not 100\%, thus the amount of hemin within hemin@ZIF-8 actually participated in the catalytic reaction should be less than the calculated amount $(2 \mu \mathrm{M})$; second, when hemin@ZIF-8 was used as the nanozyme, the color of the testing solution changed to green immediately as soon as hemin@ZIF-8 was added, thus the reaction rate should be underestimated due to the inevitable time-lag between the sample mixing and the subsequent UV-visible spectroscopy monitoring. 


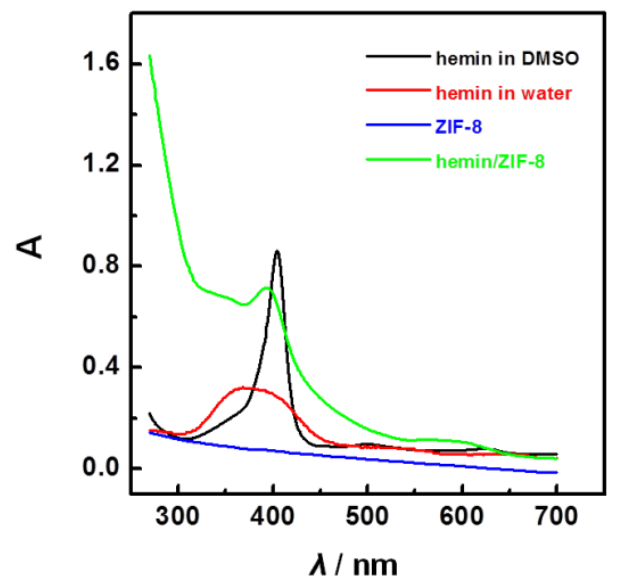

Figure S8. UV-visible spectra of free hemin dissolved in DMSO (black curve) or water (red curve), ZIF-8 (blue curve) and hemin@ZIF-8 (green curve) aqueous solutions. 


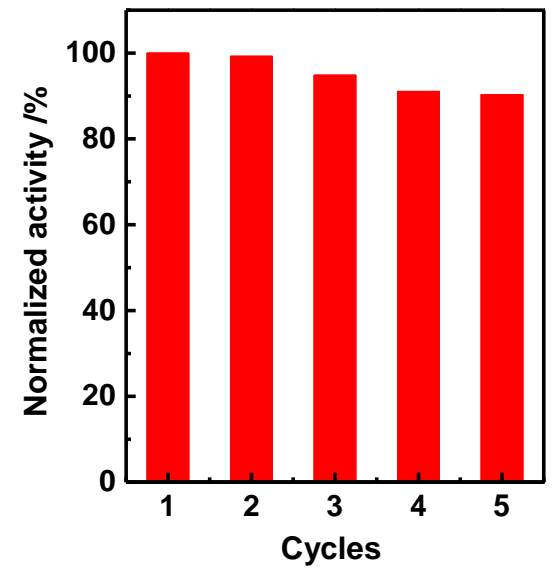

Figure S9. Normalized catalytic activities of hemin@ZIF-8 after recycling.

The hemin@ZIF-8 remained more than $90 \%$ of its original peroxidase mimic activity toward the oxidation reaction between $\mathrm{H}_{2} \mathrm{O}_{2}$ and ABTS after 5 repeating experiments (Figure S9). The slightly lowered activity may be ascribed to the inevitable loss of hemin@ZIF-8 during centrifugation or the potential leakage of the encapsulated guests from the ZIF-8 framework. 


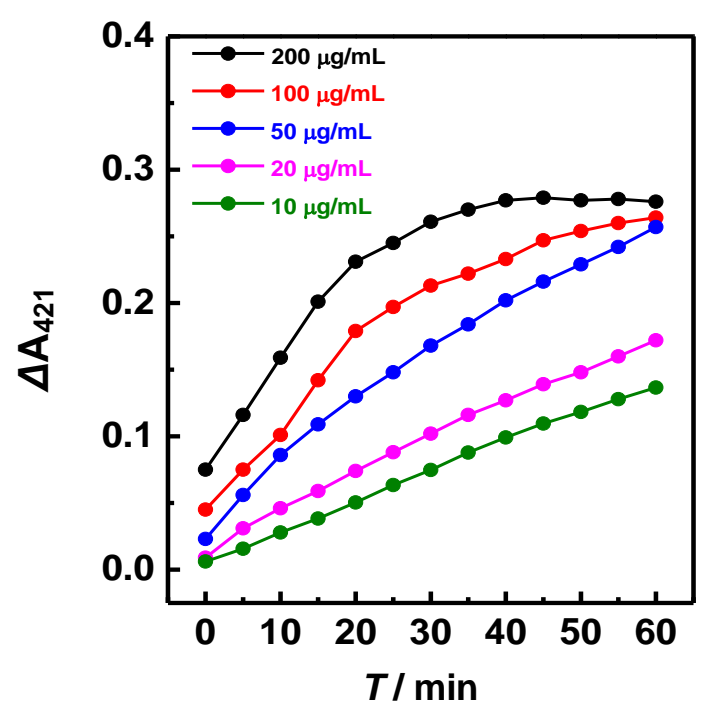

Figure S10. Kinetic plots for time-dependent $\Delta \mathrm{A}_{421}$ of $0.10 \mathrm{M}$ Tris-HCl buffer (pH 7.0) containing 250 $\mu \mathrm{M}$ glucose, $2 \mathrm{mM}$ ABTS, and different concentrations of the INAzyme. The final concentrations of the INAzyme were indicated in the figure.

The optimal concentration of the INAzyme for fast and simple colorimetric visualization of glucose was studied. To this end, different concentrations of the INAzyme were sequentially added into $0.10 \mathrm{M}$ Tris-HCl buffer ( $\mathrm{pH}$ 7.0) containing $250 \mu \mathrm{M}$ glucose and $2 \mathrm{mM}$ ABTS and the resulting solution were continuously monitored by $\mathrm{UV}$-visible absorption spectroscopy under the incubation at $37{ }^{\circ} \mathrm{C}$. As shown in Figure S10, when the concentration of the INAzyme was $200 \mu \mathrm{g} / \mathrm{mL}$, the $\Delta \mathrm{A}_{421}$ quickly reached its plateau, indicating that the cascade enzymatic reactions were completed within 40 min. Thus, $200 \mu \mathrm{g} / \mathrm{mL}$ of the INAzyme was chosen for the further studies. 


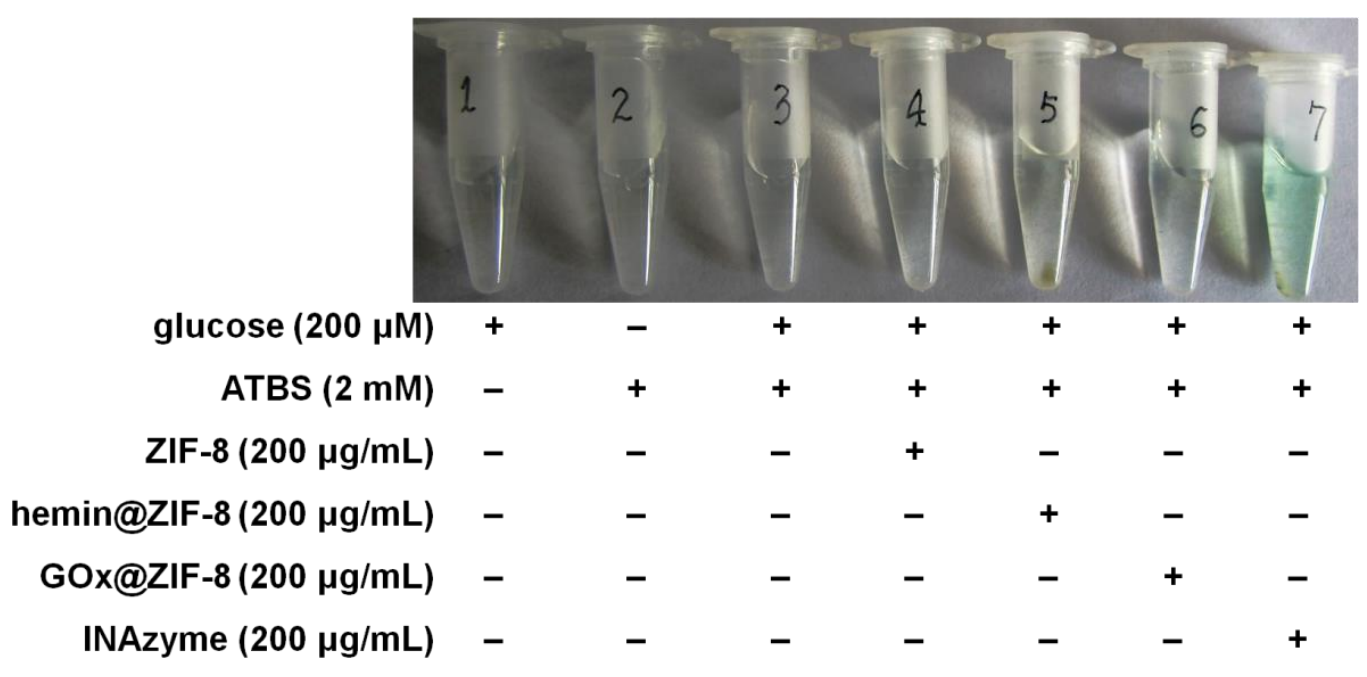

Figure S11. Typical photograph of $1 \mathrm{~mL}$ of $0.10 \mathrm{M}$ Tris- $\mathrm{HCl}$ buffer (pH 7.0) containing (1) $200 \mu \mathrm{M}$ glucose, (2) $2 \mathrm{mM}$ ABTS, (3) $200 \mu \mathrm{M}$ glucose $+2 \mathrm{mM}$ ABTS, (4) $200 \mu \mathrm{M}$ glucose $+2 \mathrm{mM}$ ABTS + $200 \mu \mathrm{g} / \mathrm{mL}$ ZIF-8, (5) $200 \mu \mathrm{M}$ glucose $+2 \mathrm{mM} \mathrm{ABTS}+200 \mu \mathrm{g} / \mathrm{mL}$ hemin@ZIF-8, (6) $200 \mu \mathrm{M}$ glucose + 2 mM ABTS + $200 \mu \mathrm{g} / \mathrm{mL}$ GOx@ZIF-8, and (7) $200 \mu \mathrm{M}$ glucose + 2 mM ABTS + 200 $\mu \mathrm{g} / \mathrm{mL}$ the INAzyme. 
a

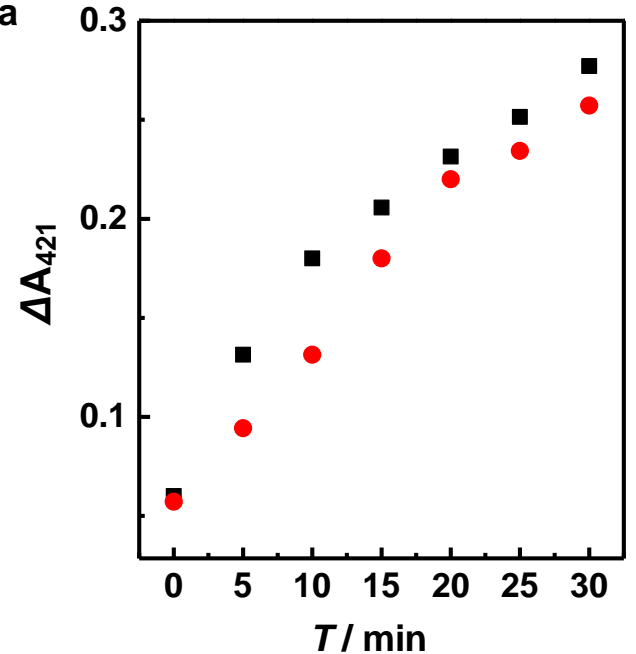

b

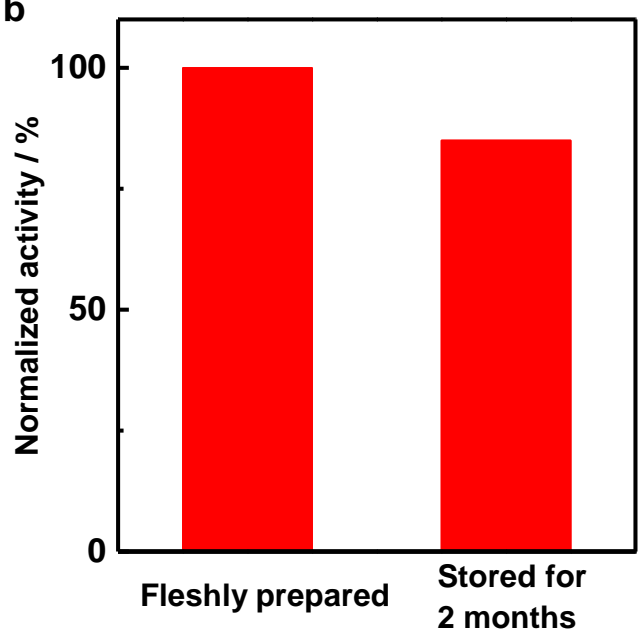

Figure S12. (a) Kinetic plots of time-dependent $\Delta \mathrm{A}_{421}$ of $0.10 \mathrm{M}$ Tris-HCl buffer (pH 7.0) containing $200 \mu \mathrm{M}$ glucose, $2 \mathrm{mM}$ ABTS, and $200 \mu \mathrm{g} / \mathrm{mL}$ fleshly prepared (•) or 2 month-old (•) INAzymes. (b) Normalized cascade enzymatic activity of fleshly prepared and 2 month-old INAzymes. 


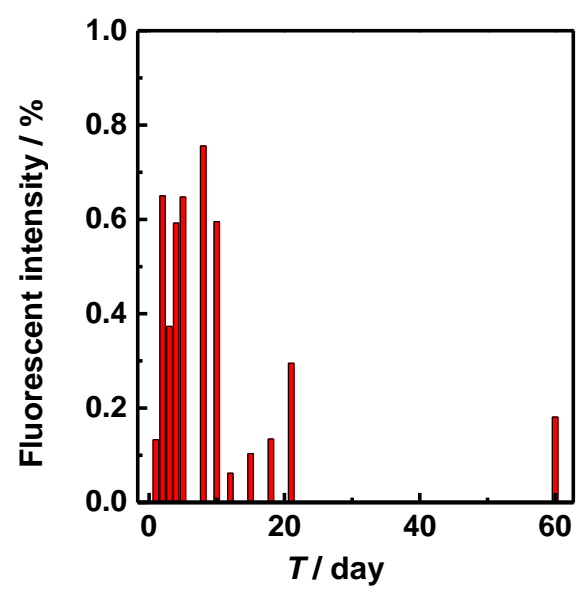

Figure S13. Normalized fluorescent intensity of leaked PPIX from GOx-FITC/PPIX@ZIF-8 over 2 months.

To evaluate the potential leakage of the encapsulated hemin and GOx from the INAzyme, the following experiments were carried out. Since both hemin and GOx are non-fluorescent, protoporphyrin IX (PPIX) and GOx-FITC conjugates were used to replace them for fabricating GOx-FITC/PPIX@ZIF-8. The supernatant of the GOx-FITC/PPIX@ZIF-8 suspension solution after centrifugation was then collected. The fluorescent responses of both PPIX and GOx-FITC in the supernatant were consecutively monitored on a fluorescent spectrometer. As shown in Figure S13, less than $0.8 \%$ of encapsulated PPIX was leaked from the ZIF-8 frameworks. More, the potentially leaked GOx-FITC was undetectable within 2 months. These results suggested that both the catalytic guests (i.e., hemin and GOx) were tightly encapsulated within the ZIF-8 frameworks. 


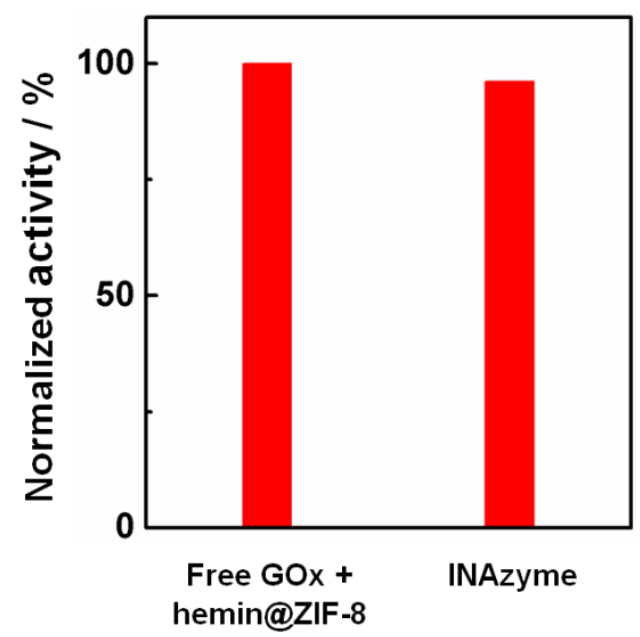

Figure S14. Normalized catalytic activity of free GOx + hemin@ZIF-8 and INAzyme, showing the encapsulation of GOx within INAzyme had little effect on its catalytic activity.

Compared with free GOx, the GOx encapsulated within the INAzyme retained more than $90 \%$ of the catalytic activity, demonstrating the excellent biomolecule-friendly environment of the ZIF-8 framework for GOx (Figure S14). More, the encapsulation of GOx within the INAzyme also endowed it with other merits such as recyclability and enhanced thermal stability relative to free GOx (Figure 3c and 4a). These results demonstrated that the nanoscaled assembly offered the designed INAzyme with synergic and enhanced properties, allowing for construction of high-performance analytical platforms. 
a

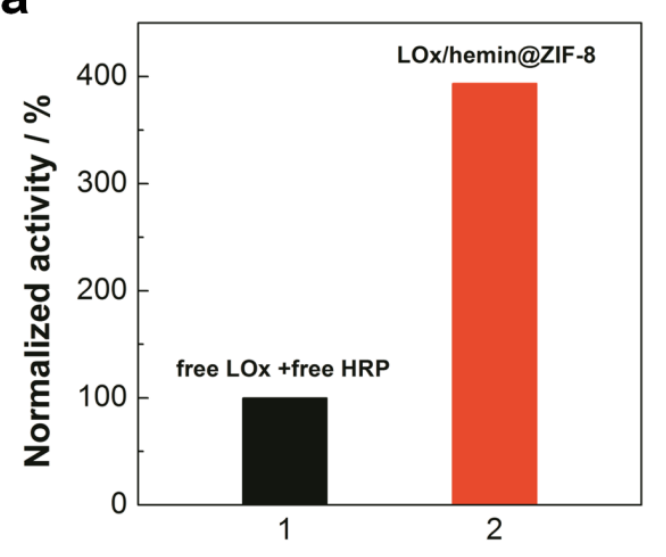

b

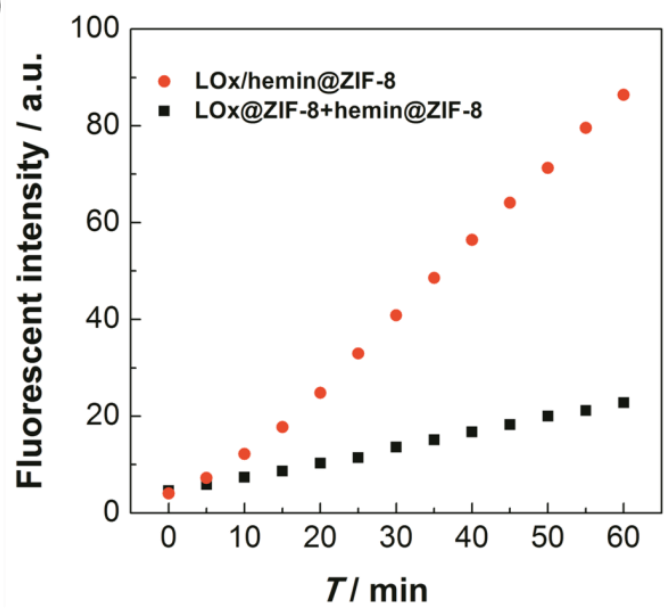

Figure S15. Nanoscale proximity effects on the catalytic activities of LOx/hemin@ZIF-8 INAzyme. (a) Normalized catalytic activities of free LOx/HRP (1) and the INAzyme of LOx/hemin@ZIF-8 (2) evaluated after $80{ }^{\circ} \mathrm{C}$ treatment, showing about $400 \%$ enhancement for the INAzyme when compared with free LOx/HRP. (b) Kinetic plots of time-dependent fluorescent intensity of $0.10 \mathrm{M}$ Tris- $\mathrm{HCl}$ buffer (pH 7.0) containing $1 \mathrm{mM}$ lactate, $155 \mu \mathrm{M}$ Ampliflu Red, and $200 \mu \mathrm{g} / \mathrm{mL}$ of the INAzyme (•) or the mixture of $200 \mu \mathrm{g} / \mathrm{mL}$ of hemin@ZIF-8 and $200 \mu \mathrm{g} / \mathrm{mL}$ of LOx@ZIF-8 ( $\mathbf{\square}$ ). 
a

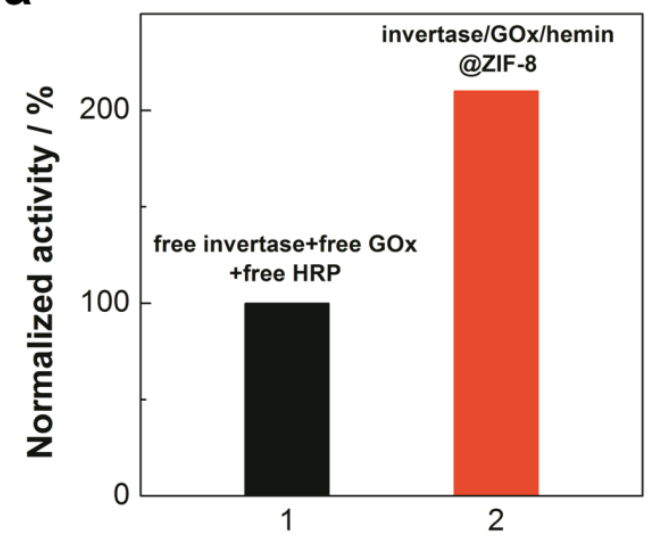

b

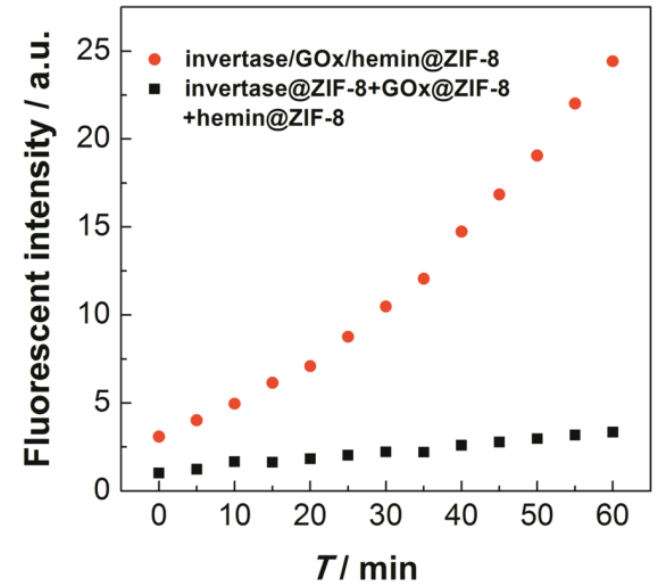

Figure S16. Nanoscale proximity effects on the catalytic activities of invertase/GOx/hemin@ZIF-8 INAzyme. (a) Normalized catalytic activities of free invertase/GOx/HRP (1) and the INAzyme of invertase/GOx/hemin@ZIF-8 (2) evaluated after $80{ }^{\circ} \mathrm{C}$ treatment, showing more than $200 \%$ enhancement for the INAzyme when compared with free invertase/GOx/HRP. (b) Kinetic plots of time-dependent fluorescent intensity of $0.10 \mathrm{M}$ Tris- $\mathrm{HCl}$ buffer ( $\mathrm{pH}$ 7.0) containing $1 \mathrm{mM}$ sucrose, 155 $\mu \mathrm{M}$ Ampliflu Red, and $200 \mu \mathrm{g} / \mathrm{mL}$ of the INAzyme (•) or the mixture of $200 \mu \mathrm{g} / \mathrm{mL}$ of hemin@ZIF-8, 200 g/mL of GOx@ZIF-8, and $200 \mu \mathrm{g} / \mathrm{mL}$ of invertase@ZIF-8 (ש).

As shown in Figure S15, when lactate oxidase (LOx) was co-assembled together with hemin inside ZIF-8, the INAzyme of LOx/hemin@ZIF-8 was obtained. As shown in Figure S15a, the LOx/hemin@ZIF-8 exhibited nearly 400\% enhancement in catalytic activity relative to free LOx/HRP after heat treatment, demonstrating the remarkable thermal stability of the INAzyme. The INAzyme of LOx/hemin@ZIF-8 also exhibited much higher catalytic efficiency when compared with the mixture of LOx@ZIF-8 and hemin@ZIF-8 (Figure S15b). More, multiple catalytic guests could be encapsulated within ZIF-8. As shown in Figure S16, invertase, which converts sucrose to glucose, was co-assembled with GOx and hemin into ZIF-8 to form the triplet INAzyme of invertase/GOx/hemin@ZIF-8. The triplet INAzyme exhibited significantly improved thermal stability (Figure S16a). It showed more than $700 \%$ faster catalysis toward the cascade reactions, relative to the separated catalysts of invertase @ZIF-8, GOx @ZIF-8, and hemin@ZIF-8 (Figure S16b). 
a

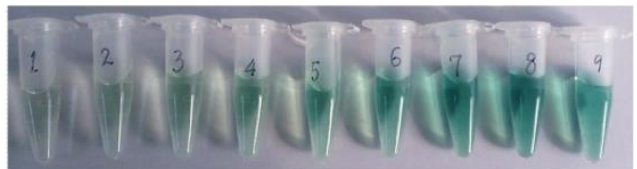

b
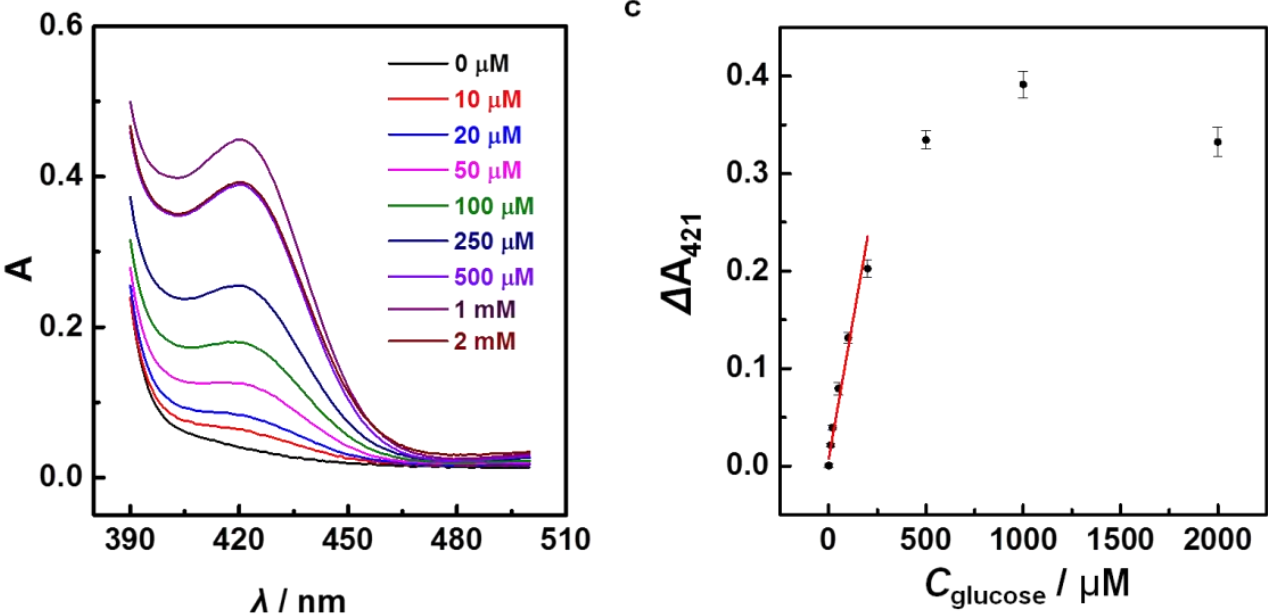

Figure S17. In vitro detection of glucose with the INAzyme. (a) Typical photograph of different concentrations of glucose in $0.10 \mathrm{M}$ Tris- $\mathrm{HCl}$ buffer ( $\mathrm{pH} 7.0$ ) containing $200 \mu \mathrm{g} / \mathrm{mL}$ INAzyme and 2 mM ABTS (from 1 to 9, the concentrations of glucose were $0 \mu \mathrm{M}, 10 \mu \mathrm{M}, 20 \mu \mathrm{M}, 50 \mu \mathrm{M}, 100 \mu \mathrm{M}$, $250 \mu \mathrm{M}, 500 \mu \mathrm{M}, 1 \mathrm{mM}$, and $2 \mathrm{mM}$, respectively). (b) UV-visible absorption spectra of different concentrations of glucose in $0.10 \mathrm{M}$ Tris- $\mathrm{HCl}$ buffer ( $\mathrm{pH} 7.0$ ) containing $200 \mu \mathrm{g} / \mathrm{mL}$ INAzyme and 2 mM ABTS. (c) Plots of $\Delta \mathrm{A}_{421}$ versus glucose concentrations. Error bars indicate standard deviations of three independent measurements. 


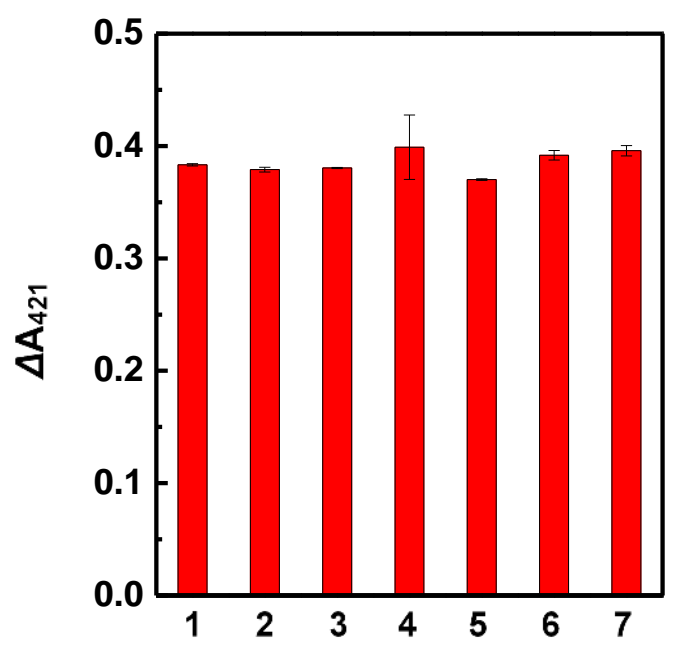

Figure S18. Selective detection of glucose with the INAzyme. Typical values of $\Delta \mathrm{A}_{421}$ of $0.10 \mathrm{M}$ Tris-HCl buffer (pH 7.0) containing $200 \mu \mathrm{g} / \mathrm{mL}$ INAzyme, $2 \mathrm{mM}$ ABTS, and $500 \mu \mathrm{M}$ glucose in the absence (1) or presence of $1 \mathrm{mM}$ lactate (2), $10 \mu \mathrm{M}$ AA (3), $10 \mu \mathrm{M}$ DA (4), $10 \mu \mathrm{M}$ DOPAC (5), $10 \mu \mathrm{M}$ 5-HT (6), or $10 \mu \mathrm{M}$ UA (7), respectively. Error bars indicate standard deviations $(\mathrm{n}=3)$. 


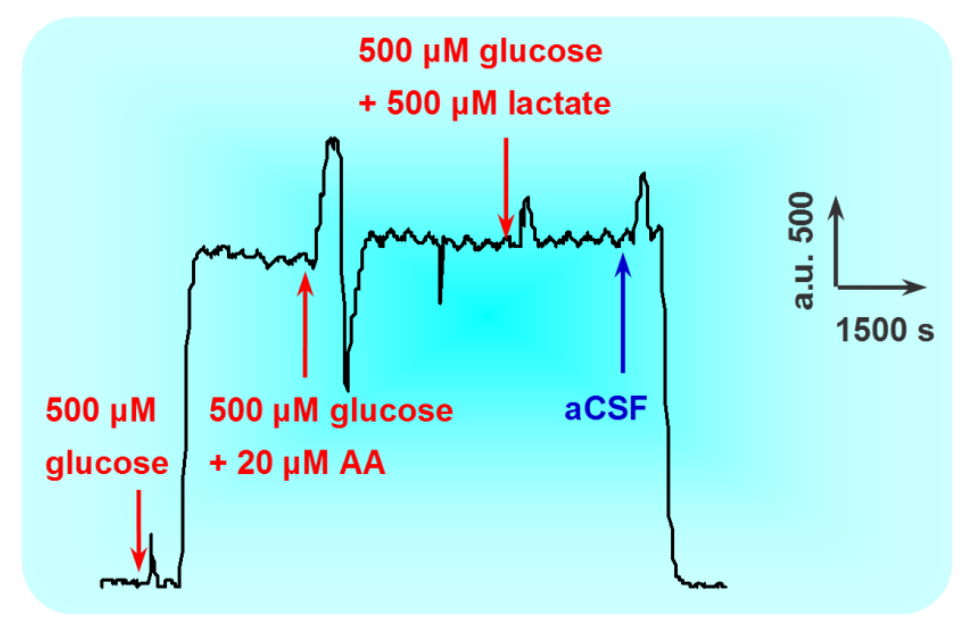

Figure S19. Typical fluorescence intensity-time curve for glucose, the mixtures of glucose and AA, and the mixtures of glucose and lactate (concentrations indicated in the figure). The response curve was recorded with the INAzyme-based integrative sensing platform (see Figure S1). 


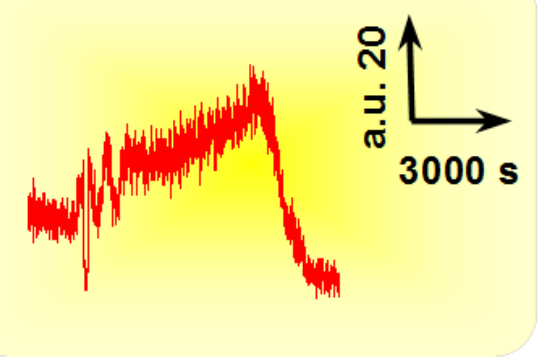

Figure S20. Typical fluorescence intensity-time curve for the microdialysate monitored on the integrative sensing platform with GOx@ZIF-8-immobilized microfluidic chip.

For hemin@ZIF-8-immobilized microfluidic chip, no elevated fluorescent response was recorded, suggesting that the physiological level of $\mathrm{H}_{2} \mathrm{O}_{2}$ present in the cerebral system could not result in a detectable signal. For GOx@ZIF-8-immobilized microfluidic chip, however, a very weak but definitely elevated fluorescent response was observed, which may be due to the slow oxidation of Ampliflu Red by the in situ formed $\mathrm{H}_{2} \mathrm{O}_{2}$ from glucose. Compared with the physiological level of $\mathrm{H}_{2} \mathrm{O}_{2}$, the concentration of the in situ formed $\mathrm{H}_{2} \mathrm{O}_{2}$ from glucose oxidation catalyzed by GOx was much higher. Even though, the recorded response on the GOx@ZIF-8-immobilized microfluidic chip was much lower than that on the INAzyme-immobilized chip, further confirming that the necessity of integration of both GOx and hemin within ZIF-8 for glucose measurements. 
Table S1. Zeta potential of ZIF-8 and ZIF-8-based nanozymes.

\begin{tabular}{llll}
\hline & ZIF-8 & hemin @ZIF-8 & GOx/hemin@ ZIF-8 \\
\hline Zeta potential / mV & 26.5 & 24.8 & 24 \\
\hline
\end{tabular}




\section{References}

(3) Gao, L. Z.; Zhuang, J.; Nie, L.; Zhang, J. B.; Zhang, Y.; Gu, N.; Wang, T. H.; Feng, J.; Yang, D. L.; Perrett, S.; Yan, X. Nat. Nanotechnol. 2007, 2, 577-583.

(7) Zheng, X.; Liu, Q.; Jing, C.; Li, Y.; Li, D.; Luo, W.; Wen, Y.; He, Y.; Huang, Q.; Long, Y.-T.; Fan, C. Angew. Chem. Int. Ed. 2011, 50, 11994-11998.

(26) Tonga, G. Y.; Jeong, Y. D.; Duncan, B.; Mizuhara, T.; Mout, R.; Das, R.; Kim, S. T.; Yeh, Y. C.; Yan, B.; Hou, S.; Rotello, V. M. Nat. Chem. $\mathbf{2 0 1 5}, 7,597-603$.

(30) Kim, C. K.; Kim, T.; Choi, I. Y.; Soh, M.; Kim, D.; Kim, Y. J.; Jang, H.; Yang, H. S.; Kim, J. Y.; Park, H. K.; Park, S. P.; Park, S.; Yu, T.; Yoon, B. W.; Lee, S. H.; Hyeon, T. Angew. Chem. Int. Ed. 2012, 51, 11039-11043.

(35) Liu, Y.; Purich, D. L.; Wu, C.; Wu, Y.; Chen, T.; Cui, C.; Zhang, L.; Cansiz, S.; Hou, W.; Wang, Y.; Yang, S.; Tan, W. J. Am. Chem. Soc. 2015, $137,14952-14958$.

(37) Li, Y. Y.; He, X.; Yin, J. J.; Ma, Y. H.; Zhang, P.; Li, J. Y.; Ding, Y. Y.; Zhang, J.; Zhao, Y. L.; Chai, Z. F.; Zhang, Z. Y. Angew. Chem. Int. Ed. 2015, 54, 1832-1835.

(41) Liu, Y.; Du, J. J.; Yan, M.; Lau, M. Y.; Hu, J.; Han, H.; Yang, O. O.; Liang, S.; Wei, W.; Wang, H.; Li, J. M.; Zhu, X. Y.; Shi, L. Q.; Chen, W.; Ji, C.; Lu, Y. F. Nat. Nanotechnol. 2013, 8, 187-192.

(47) (a) Shieh, F. K.; Wang, S. C.; Yen, C. I.; Wu, C. C.; Dutta, S.; Chou, L. Y.; Morabito, J. V.; Hu, P.; Hsu, M. H.; Wu, K. C. W.; Tsung, C. K. J. Am. Chem. Soc. 2015, 137, 4276-4279.

(47) (b) Liang, K.; Ricco, R.; Doherty, C. M.; Styles, M. J.; Bell, S.; Kirby, N.; Mudie, S.; Haylock, D.; Hill, A. J.; Doonan, C. J.; Falcaro, P. Nat. Commun. 2015, 6, 7240 . 